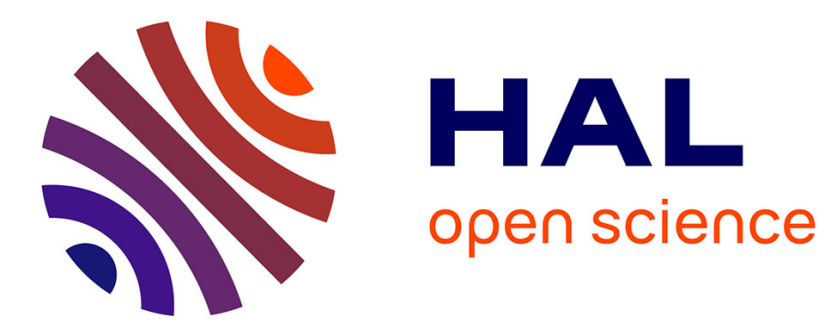

\title{
Les effets des activités auto-descriptives sur la clarté du concept de soi : le rôle modérateur des variables sociodémographiques \\ Patrick Valéau
}

\section{- To cite this version:}

Patrick Valéau. Les effets des activités auto-descriptives sur la clarté du concept de soi : le rôle modérateur des variables sociodémographiques. Psychologie du travail et des organisations, 2013, 19 (3), pp.245-262. 10.1016/S1420-2530(16)30055-3 . hal-02285934

\section{HAL Id: hal-02285934 \\ https://hal.univ-reunion.fr/hal-02285934}

Submitted on 25 Sep 2019

HAL is a multi-disciplinary open access archive for the deposit and dissemination of scientific research documents, whether they are published or not. The documents may come from teaching and research institutions in France or abroad, or from public or private research centers.
L'archive ouverte pluridisciplinaire HAL, est destinée au dépôt et à la diffusion de documents scientifiques de niveau recherche, publiés ou non, émanant des établissements d'enseignement et de recherche français ou étrangers, des laboratoires publics ou privés. 


\title{
Les effets des activités auto-descriptives sur la clarté du concept de soi : le rôle modérateur des variables sociodémographiques
}

\section{The effects of self-describing activities on self-concept clarity: the moderating effect of socio-demographic variables}

Patrick Valéau *

* IAE de la Réunion, Centre d'Economie et de Management de l'Océan Indien (CEMOI)

9 rue des Pétrels, 97417 La Montagne

patrick@valeau.com

\begin{abstract}
Résumé
Cette recherche questionne la possibilité d'induire le développement de la clarté du concept de soi (CCS) au moyen d'activités auto-descriptives. Le dispositif testretest mis en place comprenait un groupe neutre (aucune activité intermédiaire, $\mathrm{n}=40$ ), un groupe de contrôle (activité logico-cognitive, $\mathrm{n}=47$ ) et un groupe expérimental (activité auto-descriptive, $\mathrm{n}=97$ ). Les résultats montrent que l'activité auto-descriptive génère les effets les plus importants, mais que ces derniers sont plus marqués pour les individus présentant un faible niveau de CCS, ceux-ci appartenant généralement aux catégories sociodémographiques les moins valorisées. Nous interprétons ces processus en termes de « remédiation » et explorons les possibilités de mesurer et de développer en ces termes les apports des bilans de compétences.
\end{abstract}

\section{Abstract}

This research questions the possibility of inducing the development of selfconcept clarity (SCC), through a self-describing activity. A test-retest device comprises three groups: neutral (no intermediate activity), control (logicalcognitive activity), experimental (self-describing activity). The results indicate that the self-describing activity generates the largest effect, but become significantly stronger when individuals start with a low CCS, which is often the when belonging to less values socio-demographic categories. We reinterpet these process in terms of "remediation" et we explore possibilities to measure and develop professional orientation practices for this purpose. 


\section{Introduction}

La clarté du concept de soi (CCS) peut être définie comme le fait de se percevoir de façon cohérente, stable et certaine (Campbell, 1990). Brunot et Juhel (2010) proposaient de recourir à cet indicateur pour évaluer l'efficacité de "pratiques visant à enrichir la connaissance de soi » (p.1) Les bilans de compétences notamment peuvent être abordés en ces termes (Levy-Leboyer, 1993). Initialement conçus pour la gestion des carrières, cet «outil» s'est rapidement imposé comme un moyen d'accompagner des publics fragilisés par les licenciements et le chômage de longue durée (Dares, 2005). Cet article vise, tout d'abord, à apporter une première validation scientifique de la proposition de Brunot et Juhel (2010) en mesurant les effets des activités auto-descriptives sur la CCS.

Nous avons mis en place un dispositif quasi-expérimental consistant à faire passer à deux reprises, à 185 répondants, le questionnaire CCS de Campbell (1990). Entre les deux passations, nous avons introduit trois types de situation : une activité auto-descriptive (groupe expérimental, $n=97$ ), une activité cognitive (groupe neutre, $n=47$ ), aucune activité (groupe témoin, $\mathrm{n}=40$ ). Notre objectif visait ainsi à comparer les effets de reclassement des scores CCS entre le pré-test et le post-test. Ces effets étaient ensuite restitués dans le cadre d'un réseau nomologique incluant des variables sociodémographiques telles que l'âge, la qualification, le sexe et la situation d'emploi, des variables psychologiques comme l'estime de soi et le score de la CCS au pré-test, et des variables psychosociales liées à la désirabilité sociale. Ce dispositif visait à tester les quatre hypothèses suivantes :

Hypothèse 1. Les effets de reclassement observés entre le pré-test et le post-test diffèrent en fonction du type d'activités intermédiaires : les effets de reclassement des activités auto-descriptives sont supérieurs à ceux des activités logico-cognitives, eux-mêmes supérieurs à ceux observés en l'absence d'activités intermédiaires.

Hypothèse 2. Les différences entre les effets de reclassement induits par les diverses activités dépendent du niveau initial de la CCS. Les effets reclassement des répondants avec une CCS initiale faible sont supérieurs à ceux des répondants avec une CCS initiale élevée.

Hypothèse 3. Le niveau initial de la CCS dépend des données sociodémographiques des répondants.

Hypothèse 4. Les différences entre les effets reclassement induits par les diverses activités intermédiaires dépendent des données sociodémographiques des répondants. 
L'objectif plus général de cette recherche reste l'approfondissement des connaissances des phénomènes liés à la CCS. Nous pensons que le concept de clarté du concept de soi n'a pas encore reçu suffisamment d'attention dans le monde francophone, exception faite des textes de Brunot et Juhel (2010) et Pasquier et Valéau (2012). La plupart des recherches anglophones publiées depuis Campbell (1990) se sont attachées à mesurer les conséquences de la CCS, notamment sur les choix de carrières. Cette recherche se situe davantage au niveau de ses causes. L'une des principales questions, au regard notamment des problématiques relatives aux bilans de compétences, reste la "plasticité » de la CCS, autrement dit sa capacité à évoluer et la possibilité, si nécessaire, de la "réparer ». Nous commençons par une revue de littérature sur les travaux publiés sur la CCS depuis 1990 et positionnons quatre séries de conjectures relatives aux effets des questionnaires auto-descriptifs sur la CCS. Nous présentons ensuite le dispositif expérimental mis en place et les résultats obtenus. Nous discutons enfin de ce que ces derniers nous apprennent sur la CCS en proposant un modèle intégrateur rendant compte de sa nature duale entre congruence psychologique et insertion psychosociale. Nous concluons sur les applications pratiques en lien avec l'usage des activités auto-descriptives dans le cadre des bilans de compétences.

\section{Définition des concepts, résultats des recherches antérieures et conjectures}

Le concept de soi correspond à un ensemble de savoirs sur soi incluant des traits, des valeurs et des souvenirs. Il concerne des attributs physiques, des caractéristiques psychologiques ainsi que des positions identitaires (Campbell, 1990 ; Kihlstrom \& Cantor, 1983 ; Markus 1980). La clarté du concept de soi (CCS) renvoie au degré suivant lequel ces contenus sont définis de façon cohérente, claire, certaine et stable dans le temps (Campbell, 1990 ; Campbell, Trapnell, Heine, Katz, Lavallee, \& Lehman, 1996). La CCS rejoint ce qu'Erikson (1968) évoque comme un sentiment subjectif et tonique d'unité personnelle et de continuité.

Campbell (1990), Campbell et al. (1996) montrent que la CCS est fortement corrélée avec l'estime de soi $(r=0,61)$. Ces auteurs interprètent ce lien en termes d'interaction: les individus présentant une faible estime remettent facilement en question leur concept de soi face aux échecs et aux retours négatifs des autres. Plus récemment, Campbell, Assanand, et Di Paula (2003) ont identifié un lien modéré entre la CCS en tant que mesure d'unité personnelle et l'ajustement psychologique, autrement dit la capacité à faire face à des circonstances changeantes $(r=0,38)$. Les recherches montrent également que la CCS joue un rôle important dans les orientations 
de carrières. D'après Hannover et Kessels (2004), elle intervient dans les processus d'appariement avec les prototypes associés aux différentes filières $(r=0,63)$. D'après Brunot et Juhel (2010), elle participe à l'identification des profils associés aux différents métiers $(\beta=-0,49)$. La CCS apparaît ainsi comme un facteur important dans le développement personnel des individus en général et dans leur développement vocationnel en particulier.

Campbell et al. (1996) ont montré que la CCS était en partie liée à la personnalité, dépendant en particulier du névrosisme $(r=-0,23)$, de la conscience $(r=0,18)$, de l'extraversion $(r=0,12)$ et de l'agréabilité $(r=$ 0,08). Les études de Matto et Realo (2001) et Brunot et Juhel (2010) ont confirmé le rôle du névrosisme $(\beta=-0,57 ; \beta=-0,58)$ et de la conscience $(\beta$ $=0,37 ; \beta=0,28)$ sur la CCS. Cela dit, Lodi-Smith et Roberts (2010) ont, par ailleurs, mesuré des liens linéaires et curvilinéaires avec l'âge $(r=0,21)$ entre 20 et 60 ans. Cette possibilité de progression apparaît cependant en partie médiatisée par le revenu (Lodi-Smith \& Roberts, 2010). La part des variables situationnelles dans la CCS a également été mise en évidence par Pasquier et Valéau (2012), ces auteurs constatant des CCS plus élevées chez les personnes vivant en couple et chez celles ayant un emploi au moment des mesures. En résumé, si la CCS est liée à la personnalité, elle peut également progresser avec le temps en lien cependant avec les données situationnelles auxquelles les individus sont confrontés.

Partant de ces constats relatifs aux possibilités d'évolutions de la CCS en fonction des circonstances, notre recherche pose les questions suivantes : "Peut-on agir sur la CCS?»; «Peut-on créer un contexte favorable à son développement?»; "Dans quelle mesure les questionnaires auto-descriptifs peuvent-ils contribuer à ces évolutions?»Cette dernière question part des enjeux pratiques évoqués en introduction de cet article, en particulier de la proposition de Brunot et Juhel (2010) d'utiliser la CCS pour évaluer l'efficacité des pratiques visant à enrichir la connaissance de soi, ces dernières s'appuyant très souvent sur des questionnaires auto-descriptifs (Levy-Leboyer, 1993). Aucune étude ne semble encore avoir été menée à ce sujet, notre recherche constitue un premier pas dans cette direction. Nous pouvons cependant formuler un certain nombre d'hypothèses visant à relier ces questions, non seulement aux travaux antérieurs sur la CCS, mais également à différentes théories plus générales en psychologie.

Hypothèse 1. Les effets de reclassements observés entre le pré-test et le post-test diffèrent en fonction du type d'activités intermédiaires :

Les effets de reclassement des activités auto-descriptives sont supérieurs à ceux des activités logico-cognitives, eux-mêmes supérieurs à ceux observés en l'absence d'activités intermédiaires. 
Notre première hypothèse porte sur les effets de reclassement, autrement dit les modifications des scores de CCS, entre la première et la deuxième passation du questionnaire. Nous pensons que ces effets suivront un ordre croissant allant de l'absence d'activité vers l'activité autodescriptive en passant par l'activité logico-cognitive, cette progression prenant ainsi la forme d'un gradient. L'activité cognitive induirait chez les répondants un état mental plus rationnel les amenant ensuite, dans le posttest, à aborder leur concept de soi de façon plus descriptive et efficace. Les positionnements et les classements introduits dans le cadre des questionnaires auto-descriptifs constitueraient également des tâches en partie cognitives tout en portant directement sur les contenus du concept de soi. L'objectif de ces questionnaires consiste expressément à amener les répondants à expliciter et à affiner leurs points de vue à ce sujet (LevyLeboyer, 1993). Ils constituent un exemple typique de ce que Juhel et Bruno (2010) qualifient de "pratiques destinées à enrichir la connaissance de soi» (p. 1). A l'inverse, l'absence d'activité, autrement dit la simple répétition du questionnaire de mesure de la CCS devrait, en vertu de sa fiabilité, se traduire par une quasi-absence d'effets de reclassement.

Hypothèse 2. Les différences entre les effets de reclassement induits par les diverses activités dépendent du niveau initial de la CCS :

Les effets reclassement des répondants avec une CCS initiale faible sont supérieurs à ceux des répondants avec une CCS initiale élevée.

Cette deuxième hypothèse modère la première en considérant le rôle du niveau initial de CCS: l'effet de reclassement induit par les questionnaires auto-descriptifs serait proportionnel aux «manques» de clarté du concept de soi au départ éprouvés par le répondant. Il y aurait un effet d'interaction entre l'exercice proposé et le niveau initial de la CCS sur le reclassement. Eden et Aviram (1993) avaient établi un constat de cet ordre dans le cadre de leurs expériences sur les ateliers d'efficacité personnelle : les effets de ces derniers étaient plus importants sur les répondants les plus éloignés de la recherche d'emploi. L'idée serait que la marge de progression serait moindre lorsque le niveau de départ est déjà élevé, Eden et Aviram (1993) interprétant ces niveaux initialement faibles en termes de «besoin ». Nous postulons ainsi qu'une CCS faible, de façon similaire à l'estime de soi, constituerait une forme de "manque» par rapport à un niveau utile ou nécessaire pour pouvoir fonctionner normalement. Nous introduisons ce faisant la notion de remédiation.

Hypothèse 3. Le niveau initial de la CCS dépend des données sociodémographiques des répondants :

- la CCS initiale des répondants sans emploi est inférieure à celle des répondants en emploi ; 
- la CCS initiale des répondants de niveau bac ou moins est inférieure à celle des répondants de niveau supérieur au bac;

- la CCS initiale des femmes est inférieure à celle des hommes ;

- la CCS initiale des répondants moins âgés est inférieure à celle des répondants plus âgés.

Lodi-Smith et Roberts (2010) ont montré que la CCS pouvait être influencée, par exemple, par le niveau de revenu des répondants. Notre troisième série d'hypothèses prolonge ce constat en prenant en compte un certain nombre d'autres données sociodémographiques relatives à l'intégration sociale des répondants, telles que, par exemple, le fait d'avoir ou non un emploi. Durkheim (1897) évoquait l'exclusion comme un facteur de confusion anomique, Goffman (1963) relevait quant à lui des phénomènes de stigmatisation. Le regard négatif porté sur certaines catégories sociodémographiques par les catégories «dominantes», porteuses de la norme (Bourdieux \& Passeron, 1964), affecterait non seulement leur estime de soi, mais nuirait également, selon cette hypothèse, à la clarté du concept de soi. Les deux cas les plus typiques sont ceux de la qualification et de l'emploi, mais nous intégrons également dans ce cadre le sexe, dans un contexte de travail façonné autour des normes masculines (Laufer, 2004) et l'âge dans le cadre de démarrage de carrière souvent difficile (OIT, 2006). Cette exclusion et/ou cette distance par rapport aux normes dominantes ne signifient pas nécessairement une CSS systématiquement et absolument inférieure à la moyenne, mais une forme d'altération plus ou moins durable.

Hypothèse 4. Les différences entre les effets de reclassement induits par les diverses activités intermédiaires dépendent des données sociodémographiques des répondants.

- Les reclassements des répondants sans emploi sont supérieurs à ceux des répondants en emploi

- Les reclassements des répondants de niveau bac ou inférieur sont supérieurs à ceux des répondants de niveau supérieur au bac

- Les reclassements des femmes sont supérieurs à ceux des hommes

- Les reclassements des répondants les moins âgés sont supérieurs à ceux des répondants les plus âgés.

Les différentes catégories ici identifiées, les personnes sans emploi, les personnes avec un niveau inférieur au bac, les femmes et les répondants les moins âgés, tireraient un bénéfice potentiellement plus important des activités mises en place. Cette quatrième et dernière série d'hypothèses combine l'ensemble des hypothèses précédentes en testant la transitivité des relations entre les différents phénomènes impliqués: si les catégories socioprofessionnelles les moins valorisées ont généralement un faible niveau de CCS initiale, si les effets de reclassements induits par les activités auto- 
descriptives sont les plus importants et si ces effets sont plus marqués pour les individus présentant un faible niveau de CCS initiale alors les effets de reclassements induits par les activités auto-descriptives seront plus importants pour les catégories socioprofessionnelles les moins valorisées. Autrement dit, de façon plus interprétative, les effets remédiateurs des activités auto-descriptives seraient proportionnels aux effets altérateurs des circonstances sociodémographiques sur la CCS initiale.

\section{Méthodologie}

Nous présentons ici, tour à tour, les participants mobilisés dans le cadre de cette expérimentation, les questionnaires utilisés, la procédure mise en place et, enfin, les analyses effectuées sur les données ainsi obtenues.

\subsection{Participants}

L'échantillon total comportait 185 répondants adultes répartis entre les trois groupes de la manière suivante : 40 individus dans le groupe neutre, 47 dans le groupe contrôle et 98 dans le groupe expérimental. On dénombrait $50,8 \%$ d'hommes et $49,2 \%$ de femmes ; $55 \%$ avaient un niveau supérieur au baccalauréat et $45 \%$ un niveau inférieur ou égal à ce diplôme; enfin $50,3 \%$ avaient un emploi et $49,7 \%$ étaient sans. Les âges s'étendaient de 16 à 59 ans, avec une moyenne à 33 et un écart type de 11,4 .

\subsection{Instruments de mesure}

Le questionnaire utilisé pour mesurer la CCS était la « Self-Concept Clarity Scale » de Campbell de 1990, traduit par Brunot et Juhel (2010), dont la validité psychométrique, l'unidimensionnalité, la cohérence interne et la fidélité ont été contrevalidées par Pasquier et Valéau (2012). Nous avons également utilisé le R85 (ECPA, 1985), un test de raisonnement présentant essentiellement des suites logiques, numériques et littérales, à compléter. Nous avons enfin eu recours au «questionnaire de l'image de soi au travail Q-ISAT (version 2.9, Pasquier, 2011) : un questionnaire en format $Q$-sort composé de cent descripteurs de personnalité à classer par ordre de préférence et de rejet. Celui-ci produit le profil personnologique du répondant suivant les pôles positifs et négatifs du modèle à cinq facteurs : extraversion, névrosisme, agréabilité, conscience et ouverture à l'expérience.

\subsection{Procédures}

L'expérience mise en place consistait à mesurer la CCS à deux reprises: une première fois appelée "CCS1 pré-test» et, une seconde qualifiée de «CCS2 post-test ». Entre le pré-test et le post test, différentes activités étaient introduites. Les participants du groupe «neutre» passaient 
le questionnaire à deux reprises de façon consécutive alors que les participants des deux autres groupes effectuaient une tâche intermédiaire. Pour le groupe de "contrôle», il s'agissait du test de raisonnement R85. Pour le groupe expérimental, le questionnaire utilisé était le questionnaire de l'image de soi au travail (Q-ISAT, version 2.9, Pasquier, 2011). Quel que soit le groupe, aucun score n'était communiqué entre les épreuves.

\subsection{Analyses des données}

Nous avons établi l'effet de reclassement à partir de l'écart entre le score obtenu au pré-test et celui réalisé au post-test. Tel quel, cet écart pose différents types de problèmes : il est sensible aux effets de plafonnement et cumule les erreurs de mesure des deux passations. Parmi les différentes possibilités de remédier à ces défauts de fiabilité, nous avons retenu le reliable change index (RCI, indice fiable de changement) qui est un mode de calcul utilisé par certains cliniciens. Le RCI a d'abord été proposé par Jacobson, Follette et Revenstorf (1984) puis amélioré par Jacobson et Truax (1991). Il consiste à diviser la différence entre le post-test et le pré-test par la différence moyenne. Afin de limiter la tendance de la régression des scores vers la moyenne, Speer (1992) a modifié le mode calcul en y intégrant la mesure du pré-test ajusté selon la formule : «fidélité $x$ (prétest - $m$ prétest) + $m$ prétest $»$. C'est ce dernier mode de calcul que nous avons retenu en utilisant le programme développé par Defife (2004). Le RCI peut être utilisé soit comme une variable continue, soit pour distinguer des catégories en fonction d'une part de l'ampleur du changement et d'autre part du franchissement ou non de la limite entre les deux distributions considérées. On considère que le changement est fiable pour un RCI égal ou supérieur à $1,96(95 \%)$ mais selon le niveau d'exigence que le chercheur s'impose, des valeurs égales à $1,28(90 \%)$ et $0,84(80 \%)$ peuvent aussi être prises en compte.

Pour l'hypothèse 1 , les différences entre les effets de reclassement induits par les trois types d'activités intermédiaires, autrement dit les différences entre les scores RCI obtenus par les divers groupes constitués autour de ces dernières, ont d'abord été observées graphiquement, puis successivement mesurées par le F de Fisher (ANOVA) et par le t de Student (différence de moyennes pour échantillons indépendants). L'ANOVA indiquait si les différences à l'intérieur des groupes étaient moindres que celles observées entre les groupes, puis les différences de moyenne entre les groupes pris deux à deux permettaient de comparer et d'analyser plus finement les effets des différents protocoles. Pour l'hypothèse 2, nous avons calculé les moyennes des RCI pour chacune des deux modalités issues de la dichotomisation de la CCS1. Partant de là, nous avons effectué deux ANOVA distinctes comparant les différences d'effets entre les diverses épreuves, ainsi que trois différences de moyennes confrontant les RCI des 
individus présentant une CCS1 faible et ceux avec CCS1 forte, pour chacune des trois épreuves. Pour l'hypothèse 3 , nous avons calculé et comparé les moyennes de la CCS1 pour chacun des groupes établis autour des différentes variables sociodémographiques : situation d'emploi, niveau de diplôme, sexe et âge. Pour l'hypothèse 4, nous avons comparé les RCI de ces mêmes catégories en fonction des différentes épreuves dans le cadre d'une dernière série d'ANOVA.

\section{Résultats}

Nous commençons par une analyse des scores RCI en fonction des épreuves afin de voir si l'activité autodescriptive induit des effets de reclassement plus importants que l'activité logico-cognitive et que l'absence d'activité. Nous introduisons ensuite dans l'analyse la CCS initiale (CCS1), puis les variables sociodémographiques.

\subsection{Comparaison des scores RCI obtenus par les trois groupes}

Les scores RCI ont été calculés sur la base d'une fidélité égale à 0,96 correspondant à la corrélation pré-test-post-test du groupe neutre. Les valeurs introduites pour le pré-test sont $M=41,94$ et $E T=9,42$ et pour le post-test $M=42,92$ et $E T=9,08$. Les scores RCI obtenus pour l'ensemble des 185 répondants s'étendent de $-3,92$ à 4,09. Leur moyenne s'élève à 0,36 pour un écart type de 1,35. La distribution est unimodale (figure 1) et peut être considérée comme normale, le test de Kolmogorov-Smirnov donnant une valeur de $0,187(\mathrm{p}<0,01)$.

Figure 1. Moyenne des scores RCI par groupe

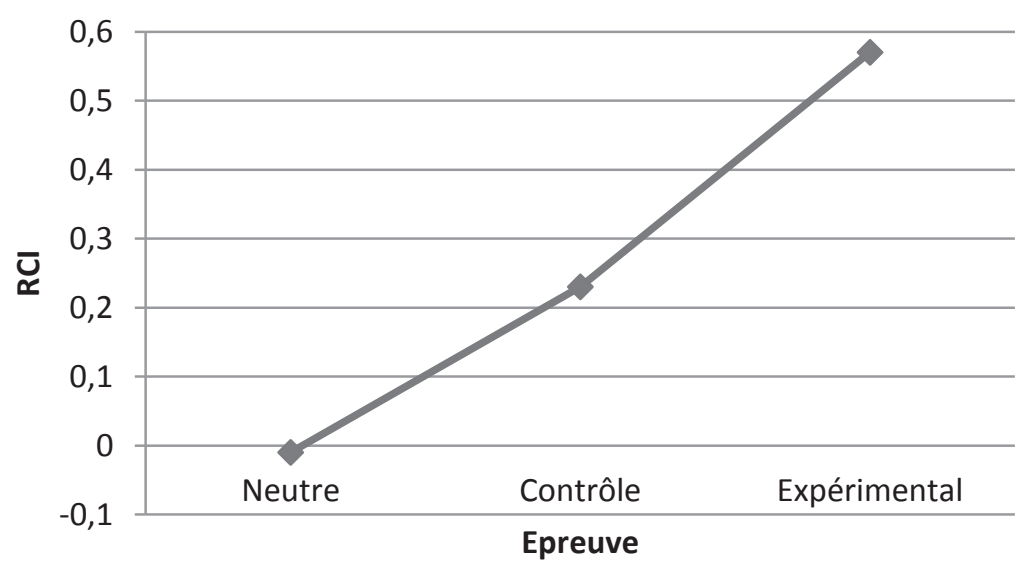


Tableau 1. Moyenne et écarts-types des scores RCI par groupe

\begin{tabular}{cccc}
\hline Groupe & $\mathrm{N}$ & $\mathrm{M}$ & $\mathrm{ET}$ \\
\hline Neutre & 40 & $-0,01$ & 1,03 \\
Contrôle & 47 & 0,23 & 1,60 \\
Expérimental & 98 & 0,57 & 1,35 \\
\hline
\end{tabular}

Tableau 2. Différences de moyennes des scores RCI entre les groupes

\begin{tabular}{ccccc}
\hline Groupe & Diff moy & $\mathrm{T}$ & Ddl & $\mathrm{P}$ \\
\hline Neutre - Contrôle & 0,24 & 0,90 & 81,56 & 0,37 \\
Contrôle - Expérimental & 0,34 & 1,34 & 83,01 & 0,18 \\
Neutre - Expérimental & $\mathbf{0 , 5 9}$ & $\mathbf{2 , 7 7}$ & $\mathbf{9 4 , 5 7}$ & $\mathbf{0 , 0 0 * *}$ \\
\hline ANOVA & $\mathbf{F}$ & $\mathbf{3 , 1 2}$ & $\mathbf{1 8 4}$ & $\mathbf{0 , 0 4 *}$ \\
\hline Neutre - Contrôle - expérimental & &
\end{tabular}

Conformément à notre première hypothèse, nous observons, dans le tableau 1 et dans la figure 1, un RCI moyen plus élevé pour le groupe expérimental $(\mathrm{m}=0,57)$ que pour le groupe contrôle $(\mathrm{m}=0,23)$, ces deux groupes avec activités intermédiaires présentant des effets de reclassement nettement plus marqués que le groupe neutre qui lui ne présente pas d'effet de reclassement $(\mathrm{m}=-0,01)$. Nous pouvons également relever des différences au niveau des écarts types: les deux groupes avec activités intermédiaires présentent des effets plus dispersés $(E T=1,35$ et $E T=1,60)$ que le groupe neutre $(E T=1,03)$. L'ANOVA pratiquée nous indique l'existence de différences significatives entre les groupes $(\mathrm{F}=3,12$; $\left.\mathrm{p}=0,047^{*}\right)$. Compte tenu de l'inclinaison de la courbe présentée dans la figure 1, cette ANOVA peut être interprétée comme une forme de relation quasi-linéaire entre les scores RCI et un gradient allant d'une absence d'activité intermédiaire vers l'activité auto-descriptive en passant par l'activité logico-cognitive. Les comparaisons des moyennes des scores RCI entre ces groupes pris deux à deux (tableau 2) confirment en partie ces analyses: la différence est très significative entre le groupe neutre et le groupe expérimental $\left(\mathrm{t}=2,77, \mathrm{p}<0,00^{* *}\right)$. Les variations entre le groupe contrôle et les deux autres n'atteignent pas, en revanche, les niveaux de significativité requis $(\mathrm{t}=0,90 ; \mathrm{p}=0,34 \& \mathrm{t}=1,34 ; \mathrm{p}=0,18)$.

\subsection{Effets de reclassement et CCS1}

Conformément à notre deuxième hypothèse, les différences entre les effets de reclassement observées d'une épreuve à l'autre apparaissent plus marquées pour les répondants présentant une CCS initialement faible que 
pour ceux possédant une CCS dès le départ relativement élevée. Les données du tableau 3 illustrées par la figure 2 indiquent même des différences significatives uniquement pour les premiers $(\mathrm{F}=3,93 ; \mathrm{p}<0,02 *)$, les différences de score RCI relevées pour les individus possédant une forte CCS1 ne sont aucunement significatives $(\mathrm{F}=0,48 ; \mathrm{p}=0,62)$. Au-delà de notre hypothèse la figure 2 et le tableau 3 montrent un effet négatif de l'absence d'épreuve pour les individus possédant une CCS initialement faible. La figure 2 montre également un croisement des courbes avec des RCI des CCS1 faibles supérieurs à ceux des CCS1 forts. Les données du tableau 4 confortent la validation de l'hypothèse 1 par le fait que les différences de reclassement entre CCS1 faibles et CCS1 fortes sont significatives uniquement dans le cadre des épreuves auto-descriptives $(\mathrm{t}=$ $2,03 ; \mathrm{p}=0,04 *)$.

Figure 2. Moyenne des scores RCI par groupe

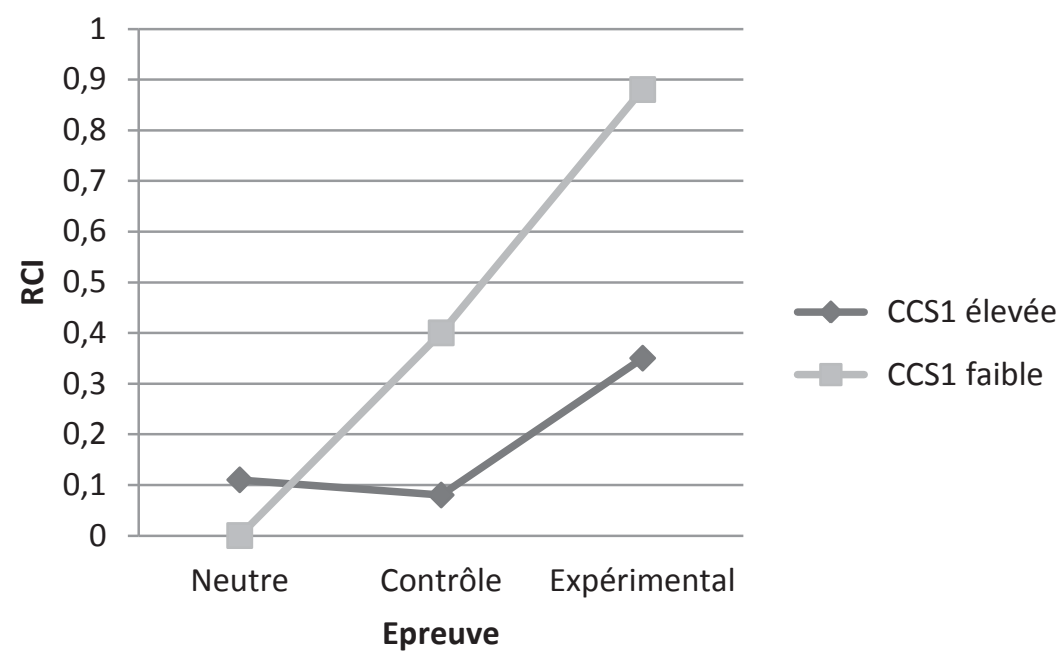

Tableau 3. Effets de reclassement en fonction du concept de soi

\begin{tabular}{|c|c|c|c|c|c|c|c|c|c|c|c|}
\hline & \multicolumn{3}{|c|}{ groupe neutre } & \multicolumn{3}{|c|}{ groupe contrôle } & \multicolumn{3}{|c|}{$\begin{array}{c}\text { groupe } \\
\text { expérimental }\end{array}$} & \multicolumn{2}{|c|}{ Statistiques } \\
\hline & $\mathrm{N}$ & M & ET & $\mathrm{N}$ & $\mathrm{M}$ & ET & $\mathrm{N}$ & M & ET & $\mathrm{F}$ & $\mathrm{P}$ \\
\hline \multicolumn{12}{|c|}{ CCS pré-test } \\
\hline Elevée & 25 & 0,11 & 0,79 & 26 & 0,08 & 1,34 & 56 & 0,35 & 1,48 & 0,48 & 0,62 \\
\hline Basse & 15 & $-0,22$ & 1,34 & 21 & 0,40 & 1,68 & 42 & 0,88 & 1,13 & 3,93 & $0,02 *$ \\
\hline $\mathrm{T}$ & & $-0,89$ & & & 0,70 & & & 2,03 & & & \\
\hline $\mathrm{P}$ & & 0,39 & & & 0,48 & & & $0,04 *$ & & & \\
\hline
\end{tabular}




\subsection{CCS1 et variables socioprofessionnelles}

Les données du tableau 4 confirment trois des quatre hypothèses relatives aux liens entre catégories socioprofessionnelles et CCS1. Les demandeurs d'emploi ont un niveau de CCS1 significativement inférieur à celui des salariés $\left(40,5<43,3 ; \mathrm{t}=2,04 ; \mathrm{p}=0,04^{*}\right)$. Le niveau des femmes apparaît moins élevé que celui des hommes $\left(40,5<43,4 ; \mathrm{t}=2,09 ; \mathrm{p}=0,03^{*}\right)$. Les plus jeunes ( $<33$ ans) ont une CCS1 plus faible que les plus âgés $(40,7<44,0$; $\left.\mathrm{t}=2,13 ; \mathrm{p}=0,03^{*}\right)$. Nos données n'ont, en revanche, pas permis d'identifier une différence significative entre les CCS1 des individus les moins qualifiés et des plus qualifiés $(40,7-43,4 ; t=1,65 ; p=0,10)$.

Tableau 4. CCS en fonction des variables sociodémographiques

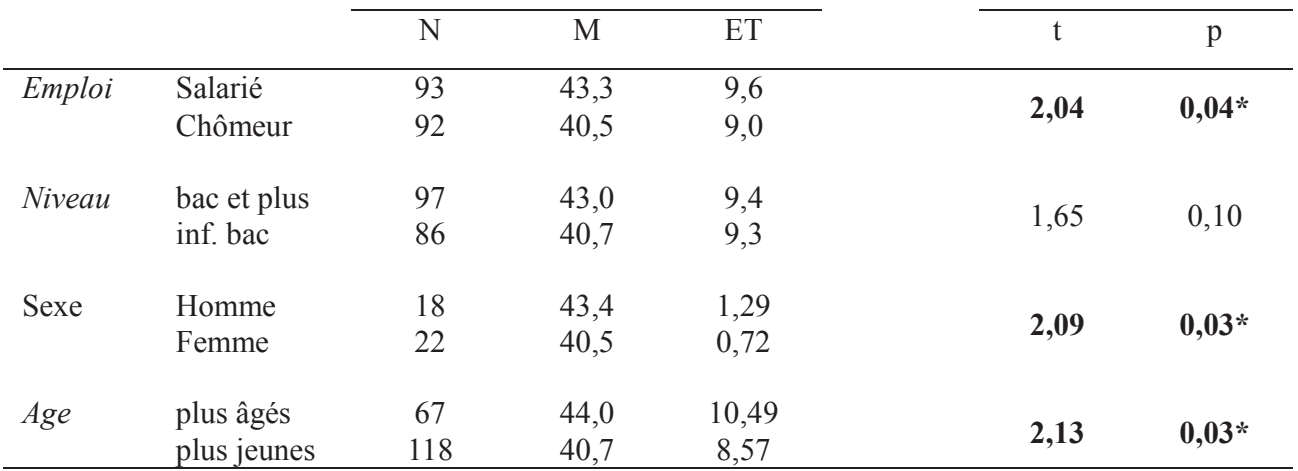

\subsection{Effets de reclassement et variables sociodémographiques}

Les scores moyens de chacune des catégories sociodémographiques augmentent en fonction du gradient d'activité (tableau 5), mais les effets de reclassement ne sont statistiquement significatifs $(p<0,5)$ que pour les répondants sans emploi; pour les répondants ne possédant qu'un niveau inférieur ou égal au baccalauréat; pour les femmes et pour les répondants les moins âgés. L'ensemble de ces résultats va dans le sens de notre quatrième série d'hypothèses : l'effet de reclassement apparaît plus marqué pour les personnes appartenant à des catégories sociodémographiques socialement moins valorisées. 
Tableau 5. Effets de reclassement en fonction des variables sociodémographiques

\begin{tabular}{|c|c|c|c|c|c|c|c|c|c|c|c|}
\hline & \multicolumn{3}{|c|}{ groupe neutre } & \multicolumn{3}{|c|}{ groupe contrôle } & \multicolumn{3}{|c|}{$\begin{array}{c}\text { groupe } \\
\text { expérimental }\end{array}$} & \multirow[b]{2}{*}{$\mathrm{F}$} & \multirow[b]{2}{*}{$\mathrm{p}$} \\
\hline & $\mathrm{N}$ & M & ET & $\mathrm{N}$ & M & ET & $\mathrm{N}$ & M & ET & & \\
\hline \multicolumn{12}{|l|}{ Emploi } \\
\hline Salarié & 21 & 0,06 & 1,16 & 22 & 0,05 & 1,44 & 50 & 0,40 & 1,46 & 0,54 & 0,30 \\
\hline & 19 & - & 0,88 & 25 & 0,38 & 1,57 & 47 & 0,76 & 1,22 & 3,61 & $\mathbf{0 , 0 3 *}$ \\
\hline $\begin{array}{l}\text { Chômeur } \\
\text { Niveau }\end{array}$ & & 0,10 & & & & & & & & & \\
\hline $\begin{array}{l}\text { bac et } \\
\text { plus }\end{array}$ & 15 & $\overline{0}, 01$ & 0,66 & 27 & 0,37 & 1,48 & 57 & 0,29 & 1,42 & 2,28 & 0,13 \\
\hline inf. bac & 25 & - & 1,21 & 20 & 0,04 & 1,54 & 41 & 0,97 & 1,16 & 6,08 & $0,03 *$ \\
\hline \multicolumn{12}{|l|}{ Sexe } \\
\hline Homme & 18 & 0,09 & 1,31 & 25 & 0,30 & 1,69 & 48 & 0,53 & 1,48 & 0,65 & 0,52 \\
\hline Femme & 22 & $\begin{array}{c}- \\
0,10\end{array}$ & 0,75 & 22 & 0,15 & 1,46 & 50 & 0,61 & 1,23 & 3,72 & $0,05^{*}$ \\
\hline $\begin{array}{l}\text { Age } \\
\text { plus } \\
\text { âgés }\end{array}$ & 19 & 0,13 & 0,76 & 12 &,- 12 & 1,90 & 36 & 0,39 & 1,50 & 0,65 & 0,52 \\
\hline $\begin{array}{l}\text { plus } \\
\text { jeunes }\end{array}$ & 21 &,- 15 & 1,22 & 35 & 0,35 & 1,34 & 62 & 0,68 & 1,26 & 3,45 & $0,03 *$ \\
\hline
\end{tabular}

\section{Discussion}

Nos résultats ont confirmé, à deux exceptions près, toutes nos hypothèses. Conformément à la première d'entre elles, les effets de reclassement observés après l'activité auto-descriptive apparaissent significativement plus importants que ceux mesurés lorsqu'aucune activité intermédiaire n'est proposée. Conformément à notre deuxième hypothèse, ces effets apparaissent plus marqués pour les répondants présentant un niveau de CCS initialement faible. Suivant l'hypothèse 3, exception faite des populations les moins qualifiées, cette faiblesse apparait, en moyenne, plus fréquente chez les populations les moins valorisées socialement. En accord avec notre quatrième hypothèse, suivant un principe de transitivité, les effets de reclassement sont plus importants chez ces populations, plus précisément, ils ne sont significatifs que pour ces dernières. Nous rassemblons à présent ces différents résultats dans le cadre d'un modèle apportant une explication contingente des effets des activités auto-descriptives sur la CCS (figure 3). En résumé, les effets des activités auto-descriptives sur la CCS sont modérés par les besoins de remédiation, ces derniers dépendant du type de public (ex. chômeurs, jeunes etc.), mais aussi de la résilience des individus face à ces situations. Nous ouvrons la discussion sur la question de la consolidation de la CCS et la durabilité des remédiations induites. Nous constatons ainsi certaines limites du dispositif mis en place et envisageons les possibilités de recherches futures. 
Figure 3. Proposition de modélisation des effets des tâches descriptives sur la CCS

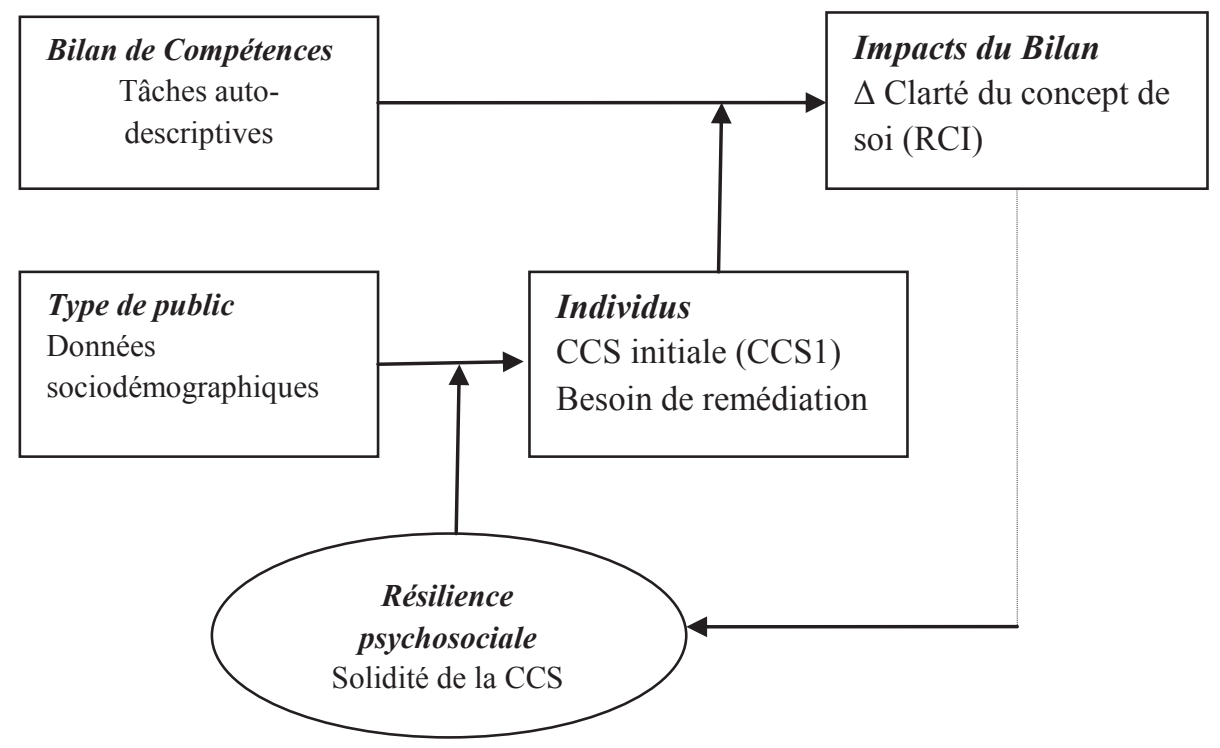

Le besoin de «remédiation » est au cœur de notre modèle, il repose, tout d'abord, par définition, la question du caractère problématique, voire dysfonctionnel d'une "trop » faible CCS. Les effets négatifs associés à une faible CCS avaient été plus explicitement évoqués, dès l'origine du concept, par Campbell (1990) : pour elle, les individus présentant cette caractéristique étaient plus perméables aux retours négatifs des autres, perdant ainsi plus facilement leurs repères. Depuis, différents effets négatifs ont été mesurés : anxiété (Stopa et al., 2010), faible capacité d'ajustement psychologique (Campbell, Assanand, \& Di Paula, 2003), difficulté à s'orienter (Brunot \& Juhel, 2010; Hannover \& Kessels, 2004). Setterlund \& Niedenthal (1993) ont mis en évidence une difficulté plus générale qu'éprouvent ces individus à se positionner socialement et prendre des décisions, par exemple pour choisir une profession ou une entreprise où postuler. De futures recherches seraient encore nécessaires pour étudier de façon plus spécifique les conséquences négatives de la CCS en termes d'employabilité, notamment lorsqu'il s'agit de reconstruire un projet professionnel ou de se présenter clairement et favorablement dans le cadre des processus de sélection. Partant de là, les contenus des bilans de compétences pourraient être mieux adaptés en fonction des besoins, avec des contenus plus psychosociaux destinés à aider la maturation du projet professionnel pour les individus avec une faible CCS et des contenus plus techniques, davantage focalisés sur les compétences acquises, pour ceux partant d'une CCS plus élevée.

Un autre apport de cette recherche concerne la fragilité de la CCS, celle-ci tendant, comme nous l'avons montré, à se dégrader à l'épreuve des 
évènements négatifs, en particulier face au chômage. Les conséquences de ce dernier sur l'estime de soi ont de longue date été soulignées (ex. Shamir, 1986). Nous considérons ici ses effets directs, non médiatisés par l'estime de soi, sur la CCS: le travail constitue un objet central d'identification psychologique et sociale, la perte de ce repère peut être source de confusion. Différentes recherches pourraient être engagées pour mesurer les temps de latence entre un licenciement et la diminution de la CCS, ainsi que le temps nécessaire à son rétablissement après avoir retrouvé un emploi. De telles données permettraient d'isoler et de mieux évaluer les apports spécifiques des bilans de compétences.

Nos résultats indiquent également que les femmes et les plus jeunes présentent un niveau de CCS moindre que les hommes et les personnes plus âgées. Différentes explications relatives à la psychologie féminine et aux problématiques de maturation liées à l'âge peuvent être avancées, mais nous pensons qu'à l'instar du chômage, le poids des jugements sociaux (Becker, 1961 ; Goffman, 1963), expliquent une partie de ces différences. La distance entre la façon dont la personne se perçoit dans cette situation et ses aspirations à se conformer à certaines normes sociales (Pasquier \& Valéau, 2011), en particulier à celles de groupes auxquels elle souhaite «s'apparier» (Brunot \& Juhel, 2010; Hannover \& Kessels, 2004; Setterlund \& Niedenthal, 1993), peut nuire à la CCS. Si une forte CCS favorise l'appariement avec les prototypes sociaux (Brunot \& Juhel, 2010 ; Hannover \& Kessels, 2004 ; Setterlund \& Niedenthal, 1993), nos résultats montrent que, réciproquement, un éloignement non désiré de ces derniers diminuerait la CCS. Ces problématiques à mi-chemin entre psychologie et sociologie, entre équilibre psychologique et intégration sociale peuvent, selon nous, être également introduites dans le cadre des bilans de compétences, l'objectif serait d'aider les stagiaires à prendre une certaine distance vis-à-vis des jugements sociaux.

Nos résultats relèvent la fragilité de la CCS mais également la possibilité de la «réparer». Nos résultats indiquent une différence de RCI très significative entre l'absence d'activité et l'activité auto-descriptive. L'ANOVA comparant les trois groupes est significative, mais les différences de moyenne ne nous ont pas permis de trancher sur la place de l'activité logico-cognitive entre l'absence d'activité et l'activité auto-descriptive. Les variations observées vont dans le sens de nos hypothèses, mais elles n'étaient pas suffisamment importantes pour valider l'existence d'effets de reclassement significatifs. De futures recherches devront étudier plus en profondeur les mécanismes sous-jacents aux remédiations observées dans le cadre des activités auto-descriptives. Parmi les pistes possibles, nous suggérons le rapprochement conceptuel de la CCS et de la « congruence» de Rogers (1961): une acceptation de soi pouvant apporter un sentiment 
d'unité, subjectivement ressenti mais toujours difficile à mesurer scientifiquement. Entre auto-description et auto-présentation de soi (Pasquier \& Valéau, 2011), les inventaires de personnalité tels que le Q-ISAT constitueraient des espaces socialement neutres, dans le cadre desquels l'individu pourrait, comme dans les entretiens non-directifs, s'exprimer et se reconstruire. Nous rejoignons Levy-Leboyer (1993) pour qui le principal enjeu des bilans demeure l'actualisation de l'image de soi : de ce fondement découlent les possibilités d'améliorations des compétences et de motivations.

L'une des principales limites de cette recherche reste l'absence de mesure de la durée des effets de remédiations induits à travers l'activité autodescriptive. Des mesures supplémentaires après quelques jours ou quelques semaines pourraient être ajoutées dans le cadre de réplications du dispositif ici initié, mais d'autres recherches pourraient aller beaucoup plus loin à travers des démarches méthodologiques «in situ» permettant de suivre des cohortes de participants à de «vrais» bilans de compétences. Les questionnaires de mesure de la CCS pourraient être intégrés, tout comme dans notre recherche, au tout début et à la toute fin de la démarche, ils pourraient être également présentés une troisième fois, quelques semaines, voire quelques mois après, afin de mesurer les effets de reclassement à moyen terme. Nous ouvrons ainsi la discussion sur la « solidité » de la CCS. Cette «solidité» permettrait de modérer les effets de situations sociodémographiques traumatisantes ou stigmatisantes sur la CCS (figure 3). Nous pensons que cette méta-propriété de la CCS pourrait être développée dans le cadre des bilans de compétences en travaillant, par exemple, sur des prises de conscience de la désirabilité sociale. Une meilleure identification des mécanismes de la stigmatisation pourrait également permettre aux participants de les désamorcer, en partie. Nous définissons, ce faisant, une forme de résilience sociale.

\section{Conclusion}

Notre dispositif méthodologique présentait l'originalité d'une démarche quasi-expérimentale reproduisant, à travers l'activité autodescriptive, une situation presque similaire à ce que Brunot et Juhel (2010) appellent les pratiques d'enrichissement de la connaissance de soi. Cette recherche confirme que la CCS constitue un outil parfaitement adapté à la problématique des bilans de compétences, elle permet de diagnostiquer de façon très synthétique les besoins de remédiations des individus, puis de mesurer les effets des pratiques mises en œuvre pour y répondre.

Notre recherche a montré qu'une simple séquence d'activité intermédiaire de nature auto-descriptive pouvait faire évoluer positivement la clarté du concept de soi. Certes, les effets de reclassement constatés restent relativement modestes, mais ils se manifestent dans le simple délai de la 
procédure de passation. Dès lors, on peut penser que des actions plus approfondies, notamment dans le cadre des bilans de compétences, pourraient avoir des effets plus marqués et plus durables. Des activités autodescriptives comme celles utilisées dans cette recherche pourraient être associées à des entretiens semi-directifs visant à ancrer les adjectifs sélectionnés dans l'expérience vécue (Rogers, 1961). Des prises de parole devant le groupe consistant à se présenter suivant ces mêmes adjectifs permettraient de les valider et de les renforcer à travers des regards extérieurs (Festinger, 1957). Partant de là, la personne peut être invitée à décliner ces caractéristiques du concept de soi dans un projet professionnel impliquant des actions concrètes dans lesquelles elle entend «s'engager ", là encore cette affirmation constitue un facteur de stabilisation et de renforcement du concept de soi (Beauvois \& Joule, 1987). L'un des éléments les plus importants introduits dans cette recherche reste la double mesure, avant et après les activités mises en place. Certaines approches des bilans de compétences accordent déjà une grande importance au travail sur l'image de soi (Levy-Leboyer, 1993). Cette mesure permet d'évaluer les progrès effectivement réalisés.

\section{Références}

Becker, H., (1963). Outsiders. Glencoe : The Free Press

Bourdieu, P., \& Passeron, J. C. (1964). Les Héritiers. Les étudiants et la culture. Paris : Minuit

Beauvois, J. L., \& Joule, R. V. (1987). Petit traité de manipulation à l'usage des honnêtes gens. Grenoble : Presses universitaires

Brunot, S., \& Juhel, J. (2010). Validation de construit d'une échelle de clarté du concept de soi. AIPTLF, Lille

Campbell, J. D., Assanand, S., \& Di Paula, A. (2003). The structure of the selfconcept and its relation to psychological adjustment. Journal of Personality, $71,115-140$

Campbell, J. D., Trapnell, P. D., Heine, S. J., Katz, I. M., Lavallee, L. F., \& Lehman, D. R. (1996). Self-concept clarity: Measurement, personality correlates, and cultural boundaries. Journal of Personality and Social Psychology, 70, 141-156

Campbell, J.D. (1990). Self-esteem and clarity of the self-concept. Journal of Personality and Social Psychology, 59, 538-549

DARES (2005). Bilans de compétences et bilans de compétences approfondis. Etudes et recherches Ministère du Travail et de l'emploi, 17, $\mathrm{n}^{\circ} 1,1-4$

DefiFe, J. (2004). Reliable change index calculator. Adelphi University, http://www.psychsystems.net/manuals/statscalculators/reliable change index calculator.xls

Durkheim, E. (1897). Le suicide, étude sociologique. Paris : PUF (1960).

Eden, D. \& Aviram, A. (1993). Self-efficacy training to speed reemployment: Helping people to help themselves. Journal of Applied Psychology, 78, 352360

Erikson, E., (1968). Adolescence et crise. La quête de l'identité. Paris : Flammarion 
Festinger, L. (1957). A theory of cognitive dissonance. Evanston, IL: Row, Peterson. Goffman, E. (1963). Stigma: Notes on the Management of Spoiled Identity. PrenticeHall.

Hannover, B., \& Kessels, U. (2004). Self-to-self prototype matching as a strategy for making academic choices. Why high school students do not like math and science. Learning and Instruction, 14, 51-67.

Jacobson, N. S., \& Truax, P. (1991). Clinical significance: A statistical approach to defining meaningful change in psychotherapy research. Journal of Consulting and Clinical Psychology, 59, 12-19.

Jacobson, N. S., Follette, W. C., \& Revenstorf, D. (1984). Psychotherapy outcome research: Methods for reporting variability and evaluating clinical significance. Behavior Therapy, 15, 336-352.

Kihlstrom, J. F., \& Cantor, N. (1983). Mental representations of the self. In L. Berkowitz (Ed.). Advances in experimental social psychology, 17, 1-47. San Diego, CA: Academic Press.

Laufer, J. (2004). Femmes et carrières : la question du plafond de verre, Revue Française de Gestion, 151, 117-127.

Levy-Leboyer, C. (1993). Le bilan de compétences. Paris : Edition d'Organisations.

Lodi-Smith, J., \& Roberts, B. W. (2010). Getting to know me: social role experiences and age differences in self-concept clarity during adulthood. Journal of personality, 78, 383-410.

Markus, H. (1980). The self in thought and memory. In D. M. Wegner \& R. R. Vallacher (Eds.). The self in social psychology (pp. 102-130). New York: Oxford University Press.

Matto, H., \& Realo, A. (2001). The Estonian self-concept clarity scale: psychometric properties and personality correlates. Personality and Individual Differences, 30, 59-70.

OIT (2006). Tendances mondiales de l'emploi des jeunes. Genève : OIT.

Pasquier, D. (1985). R85. Paris: ECPA.

Pasquier, D. (2011). Questionnaire d'image de soi au travail - QISAT -, version 2.9. Nangis : Avenir \& Entreprise.

Pasquier, D., \& Valéau, P. (2011). Adhésion à la norme d'internalité et clairvoyance normative : un passage par les contingences de la socialisation. Psychologie $d u$ Travail et des Organisations, 17, 1-26.

Pasquier, D., \& Valéau, P. (2012). Clarté du concept de soi et distance socionormative, AIPTLF, Lyon.

Rogers, C. R. (1961). On becoming a person, Houghton : Miffin Company

Setterlund, M. B., \& Niedenthal, P. M. (1993). Who am I? Why am I here? Selfesteem, self-clarity, and prototype matching. Journal of Personality \& Social Psychology, 65, 769-780.

Shamir, B. (1986). Self-esteem and the psychological impact of unemployment, Social Psychology Quarterly, 49, 6-72.

Speer, D. C. (1992). Clinically significant change: Jacobson and Truax (1991) revisited. Journal of Consulting and Clinical Psychology, 60, 402-408.

Stopa, L., Brown, M. A., Luke, M. A., \& Hirsch, C. R. (2010). Constructing a self: the role of self-structure and self-certainty in social anxiety. Behaviour Research and Therapy, 48, 155-165. 